\title{
Impacto da pandemia COVID-19 na dinâmica do núcleo de hemoterapia de um hospital universitário
}

Impact of the COVID-19 pandemic on the dynamics of a university hospital's hemotherapy center Impacto de la pandemia COVID-19 sobre la dinâmica de um centro de hemoterapia em uno hospital universitario

\begin{abstract}
RESUMO
Objetivo: Descrever ameaças e oportunidades diante da pandemia de COVID-19 no Núcleo de Hemoterapia do Hospital Universitário Pedro Ernesto. Método: estudo descritivo com distribuição de frequência de candidatos à doação de sangue antes (2015-2019) e após declaração da pandemia COVID-19 (março/2020), e descrição de estratégias para manutenção do estoque de hemocomponentes. Resultados: houve redução de $25 \%$ no número de candidatos à doação entre os dias 2 e 16 de março de 2020. A implantação de diversas atividades de promoção à doação de sangue aumentou em $20 \%$ a média de doadores/dia em março/2020. Observou-se aumento de $24 \%$ no número de candidatos à doação em março/2020 ( $n=536$ ) comparado a março/2019 ( $n=431$ ). Conclusão: Diante de uma nova doença, de caráter pandêmico, temos mais perguntas que respostas. É necessário que mais centros compartilhem suas estratégias para a garantia de sangue em momentos de crise.
\end{abstract}

DESCRITORES: Covid-19; Pandemia; Hemoterapia; Saúde Pública.

\section{ABSTRACT}

Objective: describe threats and opportunities in the face of the COVID-19 pandemic at the Pedro Ernesto University Hospital Hemotherapy Center. Method: descriptive study and quantification of blood donation candidates before (2015-2019) and after the declaration of the COVID-19 pandemic (March/2020) describing strategies to safeguard blood supply. Results: there was a 25\% reduction in the number of donation candidates between March 2nd and 16th, 2020. The implementation of several promotional initiatives increased the donor per day average in March 2020 by $20 \%$. There was a $24 \%$ increase in the number of donation candidates in March 2020 ( $n=536$ ) compared to March 2019 ( $n=431$ ). Conclusion: In the face of a new disease, of a pandemic character, we have more questions than answers. It is necessary that more centers share their strategies for guaranteeing blood in times of crisis.

DESCRIPTORS: Covid-19; Pandemic; Hemotherapy; Public Health.

\section{RESUMEN}

Objetivo: Describir las amenazas y oportunidades frente a la pandemia de COVID-19 en el Centro de Hemoterapia del Hospital Universitario Pedro Ernesto. Método: estudio descriptivo para cuantificar el numero de candidatos a donaciónes de sangre antes (2015-2019) y después de la declaración de la pandemia COVID-19 (marzo/2020) y describir estrategias para salvaguardar el suministro de sangre. Resultados: hubo una reducción del 25\% en el número de candidatos para donación entre el 2 y el 16 de marzo de 2020. La implementación de varias iniciativas promocionales aumentó el numer de donaciones/día promedio en marzo/2020 en un $20 \%$. Hubo un aumento del $24 \%$ en el número de candidatos a donar em marzo/2020 ( $n=536)$ en comparación con marzo/2019 ( $n$ = 431). Conclusión: Ante una nueva enfermedad, de carácter pandémico, tenemos más preguntas que respuestas. Es necesario que más centros compartan sus estrategias para garantizar el subministro en tiempos de crisis.

DESCRIPTORES: Covid-19; Pandemia; Hemoterapia; Salud Pública.

RECEBIDO EM: 25/04/2020 APROVADO EM: 26/04/2020

\section{Flavia Miranda Gomes de Constantino Bandeira}

Médica. Hematologista e Hemoterapeuta. Professora Adjunta do Departamento de Medicina Interna da Faculdade de Ciências Médicas, Universidade do Estado do Rio de Janeiro. RJ/Brasil. https://orcid.org/0000-0002-9499-7520

\section{Jennifer Ribeiro da Cunha}

Médica. Hematologista e Hemoterapeuta. Universidade do Estado do Rio de Janeiro. RJ/Brasil. https://orcid.org/0000-00016725-8944 


\section{Samantha Veloso Baião}

Enfermeira. Especialista em Estratégia de Saúde da Família. Especialização em Hemoterapia em curso. Universidade do Estado do Rio de Janeiro. RJ/Brasil. https://orcid.org/0000-0002-6730-6392

\section{Cristiane Magalhães Costa}

Enfermeira. Mestre em Saúde da Família. Especialista em Hemoterapia, Saúde do Trabalhador e Gestão Pública. Universidade do Estado do Rio de Janeiro. RJ/Brasil. https://orcid.org/0000-0001-5927-6528

\section{Regina Márcia Rangel de Oliveira}

Assistente Social do Banco de Sangue Herbert de Souza, Hospital Pedro Ernesto-HUPE, Universidade do Estado do Rio de Janeiro. RJ/Brasil. https://orcid.org/0000-0002-6920-1164

\section{Kallic Borba Fonseca}

Médico. Hematologista e Hemoterapeuta. Universidade do Estado do Rio de Janeiro. RJ/Brasil. https://orcid.org/0000-00020338-1705

\section{Tatiana de Araujo Eleuterio}

Enfermeira. Doutora em Epidemiologia e Saúde Pública. Sanitarista. Especialista em Saúde da Família. Professora Adjunta do Departamento de Enfermagem em Saúde Pública da Faculdade de Enfermagem, Universidade do Estado do Rio de Janeiro. RJ, Brasil. https://orcid.org/0000-0002-8043-2350

\section{INTRODUÇÃO}

A cidadede Wuhan, na província de Hubei, na China, despontou como epicentro de uma pneumonia grave causada por um novo coronavírus em dezembro de 2019. Em janeiro de 2020 já havia relato de milhares de pessoas acometidas em diversas cidades da China e do mundo ${ }^{(1,2)}$. No dia 25 de fevereiro de 2020 , foi confirmado no Brasil o primeiro caso de infecção pelo novo coronavírus (SARS-CoV-2). As primeiras notificações, inicialmente restritas ao estado de São Paulo, compreendiam casos autóctones entre pessoas com histórico de viagem a países com transmissão comunitária do vírus. Em 11 de março deste ano, a Organização Mundial da Saúde (OMS) declarou a COVID-19 como pandemia ${ }^{(3)}$ e, desde 16 de março, o governo brasileiro e as unidades da federação têm enfrentado a mesma com medidas de mitigação, tendo em vista a transmissão comunitária em todo o país, reforçando as orientações de distanciamento social que, por sua vez, impactaram direta e negativamente nos estoques dos bancos de sangue, em todo o território nacional ${ }^{(3-10)}$.

Até o dia 06 de abril de 2020, haviam sido confirmados 1.210 .956 casos de infecção pelo novo Coronavírus (COVID-19) e 67.594 óbitos no mundo, com taxa de letalidade de $5,6 \%^{(11)}$. No Brasil, eram confirmados 12.056 casos de COVID-19 e 553 óbitos, com taxa de letalidade de $4,6 \%{ }^{(12)}$.

Não havendo, a princípio, implicação direta da fisiopatologia da COVID-19 no aumento da necessidade de suporte transfusional, outras doenças de prevalência considerável no país seguem em curso, assim como cirurgias de urgência, cirurgias cardíacas e oncológicas, entre outras. A iminente redução no número de doações de sangue tornou-se uma preocupação de ordem mundial e nacional, podendo ser afetada não apenas pelo isolamento social imposto por diversos governos, como também pela temporária inaptidão de doadores, seja por passado de COVID-19, seja por procedência de região acometida pela pandemia.

Considerando o fato do Hospital Universitário Pedro Ernesto (HUPE), unidade de saúde da Universidade do Estado do Rio de Janeiro (UERJ), ter sido indicado pela Secretaria de Estado de Saúde para atuar como um dos centros de referência para assistência aos casos de COVID-19, surgiu um desafio para gestores e responsáveis pela manutenção do estoque de hemocomponentes, que deveriam priorizar o distanciamento social e, paralelamente, estimular que doadores sigam comparecendo ao serviço para doações regulares.

Antecipando-se à crise de abastecimento e manutenção de estoque de hemocomponentes, o Núcleo de Hemoterapia do Hospital Universitário Pedro Ernesto (NHHUPE) buscou avaliar ameaças e oportunidades para o enfrentamento da pandemia de COVID-19, no tocante à manutenção da assistência hemoterápica no HUPE.
Objetivou-se refletir sobre ameaças e oportunidades diante da pandemia de COVID-19 e seu potencial impacto nas doações de sangue no Núcleo de Hemoterapia do Hospital Universitário Pedro Ernesto e avaliar o impacto da implantação de estratégias de captação de doador em momento de crise.

\section{METODOLOGIA}

Avaliação quantitativa, descritiva e comparativa do número de candidatos à doação de sangue, antes e após a declaração da pandemia COVID-19, obtidos a partir do sistema informatizado utilizado rotineiramente no serviço para avaliação de indicadores de performance (Sistema Hemote Plus - SOFIS Informática Ltda.). O período de análise compreendeu os anos de 2015 a 2019 para comparação histórica com o mês de março de 2020, quando da declaração da pandemia COVID-19 pela OMS. Descrição das estratégias de promoção à doação, com análise de ameaças e oportunidades diante da pandemia. Tais análises foram realizadas a partir de base de dados secundários do serviço e constituem um recorte de projeto de pesquisa submetido ao Comitê de Ética em Pesquisa do Hospital Universitário Pedro Ernesto (CEP/UERJ), parecer n. ${ }^{\circ}$ 3.971.133, CAAE: 30649020.1.0000.5259, em respeito às disposições da Resolução CNS n. ${ }^{\circ} 466 / 2012$ sobre pesquisas envolvendo seres humanos. 


\section{RESULTADOS}

A média histórica de candidatos à doação no

NHHUPE nos últimos 5 anos é de 20 candidatos/dia, totalizando 25.300 candidatos entre 2015a2019(Figura 1).O índice defracionamen-
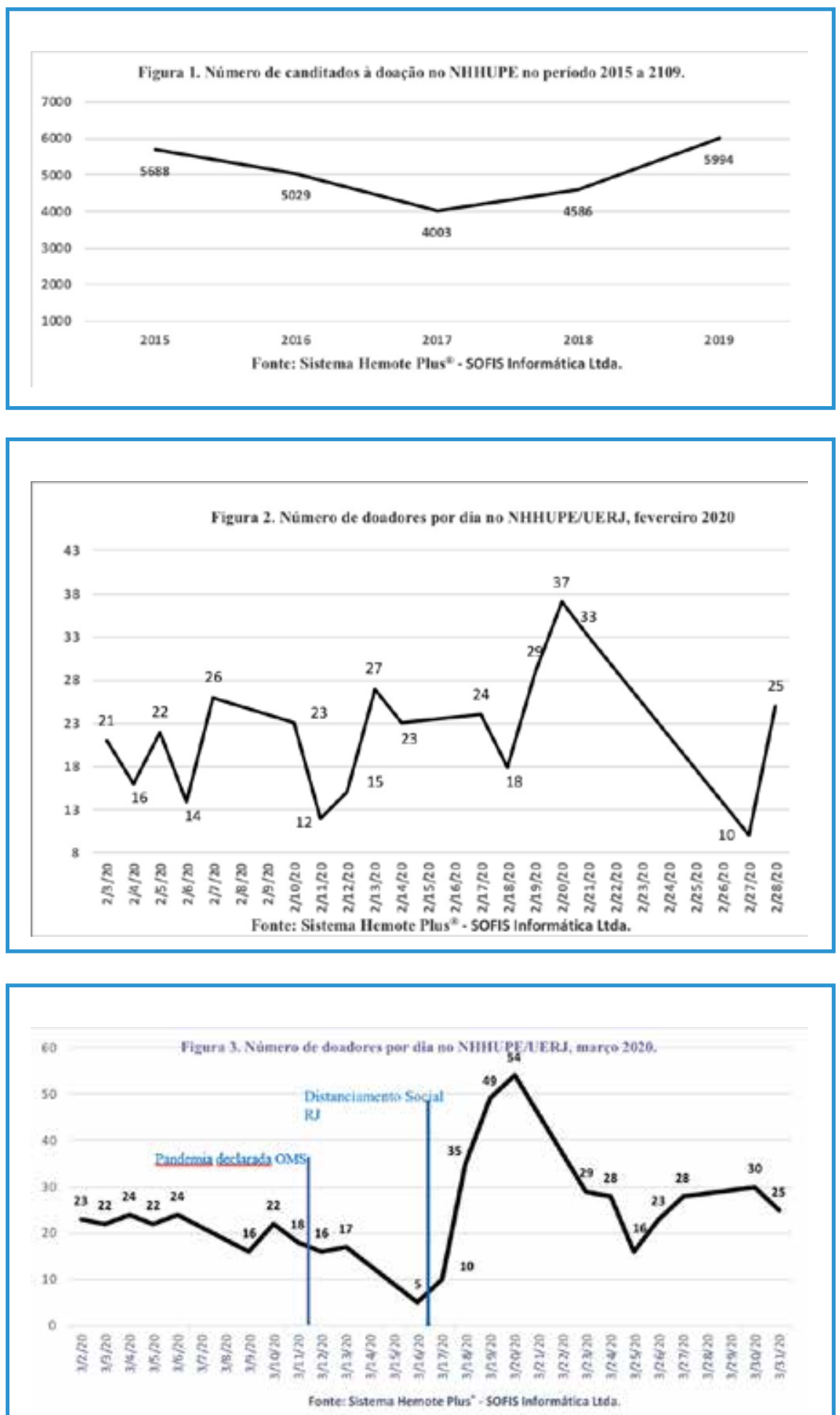

to no período foi de $2,8($ meta $=3$ ), com produção média anual de 10.814 hemocomponentes.

Nos primeiros dois meses de 2020, o NHHUPE atendeu 896 doadores, com média de 24/dia, entre doadores de sangue total e de plaquetas. As Figuras 2 e 3 mostram a evolução diária do número de doadores, nos meses de fevereiro e março de 2020.

Em fevereiro de 2020, compareceram para doação 375 candidatos, com média de 22 doadores/dia, enquanto em março/2020 foram 536 candidatos, perfazendo uma média diária de 24,4 doadores/dia. Comparando a média histórica de 20 candidatos à doação/dia, observou-se aumento de $20 \%$ na média de doação/dia em março/2020. Observou-se também aumento de $24 \%$ no número de candidatos à doação em março de 2020 ( $\mathrm{n}=536)$ quando comparado a março/2019 $(n=431)$ (Figura 4). Considerando a média histórica de 20 candidatos à doação/dia, observou-se uma queda de $25 \%$ no número de candidatos à doação entre os dias 2 e 16 de março de 2020, sendo o ápice desta queda o dia 16 de março de 2020, primeiro dia útil após o Governo do Estado do Rio de Janeiro publicar o Decreto n.o 46.966 de 11/03/2020, publicado em $13 / 03 / 2020$, e que dispunha sobre as medidas para enfrentamento da emergência de saúde pública de importância internacional decorrente do coronavírus, edava outras providências (Figura3).

\section{DISCUSSÃO}

O HUPE é o hospital universitário da Universidade do Estado do Rio de Janeiro (UERJ). Trata-se de um hospital de média e alta complexidade, com cerca de 450 leitos, atuando em diversas frentes de ensino, pesquisa e extensão, sendo o Núcleo de Hemoterapia HUPE (NHHUPE) um de seus cenários de prática. O NHHUPE conta com equipe de profissionais da saúde formada por docentes, médicos, enfermeiros, assistentes sociais, biólogos, farmacêuticos, técnicos de enfermagem e de laboratório, pessoal de apoio administrativo, apoio à limpeza e higiene, encaminhador e segurança, integrantes da dinâmica do ciclo do sangue neste hospital.

A agência transfusional (AT) do NHHUPE, localizada dentro do hospital, é responsável pela rotina pré-transfusional e expedição de hemocomponentes para os pacientes cirúrgicos, 


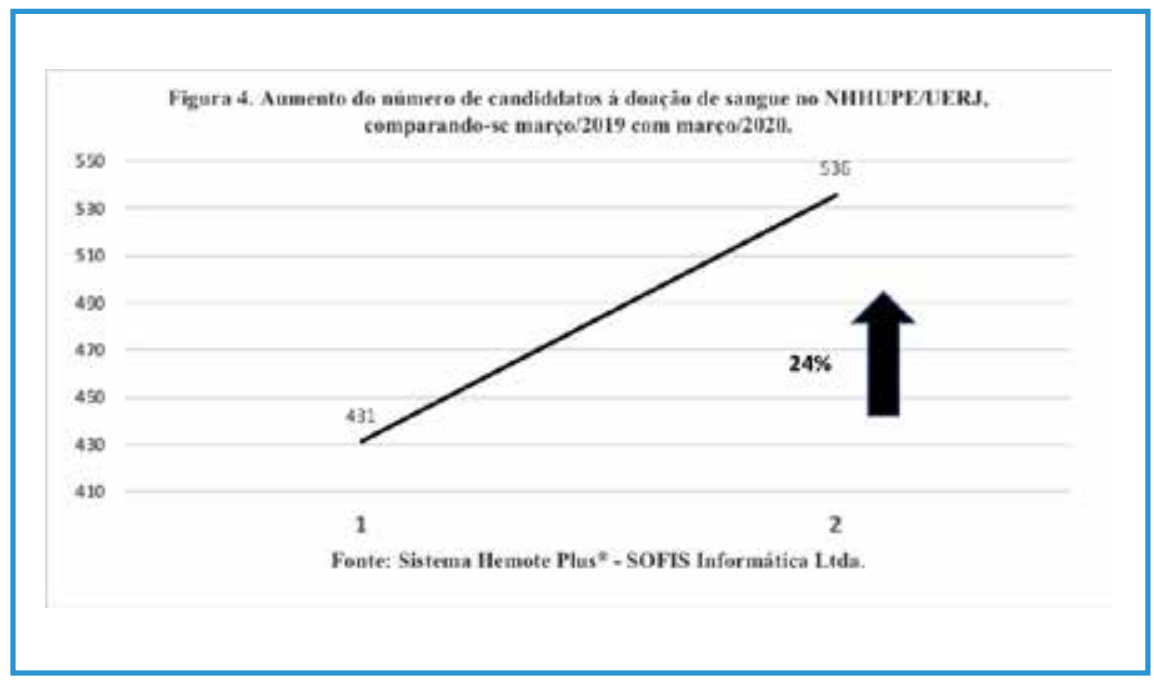

clínicos e aqueles em atendimento ambulatorial. A rotina cirúrgica no HUPE, compreende média de trinta (30) procedimentos/dia, 50\% dos quais são de médio e grande porte.

A rotina de promoção à doação de sangue é liderada por uma enfermeira e uma assistente social, com apoio da equipe do setor e das assessorias de comunicação do HUPE (COMHUPE) e da UERJ (COMUNS), TVUERJ, rádio UERJ, corpo docente e discente da universidade. Ações, tais como sensibilização sobre a importância da doação de sangue junto a profissionais do HUPE, pacientes e familiares; parcerias com instituições e empresas; articulação com setores da universidade; promoção a doação via contatos telefônicos, e-mail, mídias sociais e campanhas de doação alusivas às datas importantes do calendário anual, são algumas que visam motivar e fidelizar o doador de sangue.

O início do ano letivo é a época em que a equipe de captação, em parceria com o projeto de extensão universitária da Faculdade de Enfermagem da UERJ, centros e diretórios acadêmicos, intensifica campanhas de alcance à comunidade através de mídia e redes sociais. $\mathrm{O}$ NHHUPE, em parceria com o projeto de extensão da Faculdade de Enfermagem intitulado "Sangue: perdendo o medo garantindo a vida", havia programado uma agenda de atividades para que calouros de sete cursos de graduação da UERJ pudessem no início do ano letivo comparecer ao núcleo para se candidatar à doação de sangue e medula óssea. Uma conversa sobre condições básicas à doação de sangue e sensibilização de alunos para este evento, aconteceu no dia 03 de março de 2020, em auditório da UERJ envolvendo alunos dos cursos de Direito, Nutrição e Física da universidade, conforme publicado em página do Facebook "Projeto Sangue: vencendo o medo garantido a vida" e do Instagram “@projetosangueuerj” (13).

A campanha de doação de sangue, agendada por grupos de alunos, teria início em 16 de março de 2020. Contudo, em 13/03/2020, foi publicado o Decreto n.o 46.966 de 11/03/2020(4) pelo Governo do Estado, dispondo as medidas de enfrentamento ao SARS-CoV-2, dentre as quais destacavam-se a necessidade da implementação de medidas de distanciamento social e suspensão das atividades em escolas e universidades. A Reitoria da UERJ, em 13/03/2020, conforme nota em sua página oficial na Internet ${ }^{(14)}$ e de acordo com o Ato Executivo de Decisão Administrativa (AEDA) n.o 13/Reitoria/2020 $0^{(15)}$, suspendeu as aulas e demais atividades acadêmicas presenciais, inicialmente, por 15 dias, a partir de 16 de março de 2020.

Diante deste contexto, para que não houvesse aglomeração de pessoas no NHHUPE e respeitando as medidas de distanciamento social, a campanha "Trote Solidário" foi cancelada, ao passo que a direção do HUPE iniciava o plano de contingência para o enfrentamento da pandemia, cancelando cirurgias eletivas, mantendo ativas as cirurgias cardíacas, oncológicas e as de urgência. Foi ainda garantido o acesso a pacientes graves e àqueles com doenças hematológicas, oncológicas, hemodiálise, considerados como urgência.

Considerando a Portaria MS n.o 428, de 19 de março de $2020^{(5)}$, a pandemia tem provocado impacto nos processos de trabalho e na vida dos profissionais que atuam no serviço, pontualmente provocando o afastamento de colaboradores e servidores de suas atividades laborais. A garantia de equipamentos de proteção individual (EPI) e o cumprimento de regras de biossegurança e boas práticas passaram a ser requisitos fundamentais para o bom funcionamento do serviço e manutenção da integridade física dos profissionais. Fazia-se necessário também, o estabelecimento de um plano de contingência para o absenteísmo, causado pelos afastamentos de colaboradores, por questôes legais e eventuais confirmações ou suspeitas de contaminação por SARS-CoV-2. Vale ressaltar a sensação de "medo" e "temor" enfrentada por toda a equipe da saúde, reforçando também a necessidade de apoio emocional a todos os profissionais envolvidos nesta linha de frente no enfrentamento à pandemia.

Tendo em vista o exposto, o NHHUPE precisava se reinventar e, de acordo com as orientaçóes de autoridades sanitárias do país, do estado e entidades científicas, manter abertas as portas para doadores, reforçando as regras de higiene, distanciamento social, proteção de doadores e colaboradores, sem esquecer que as medidas de distanciamento social e quarentena, poderiam impactar negativamente $\mathrm{o}$ estoque de hemocomponentes e a assistência hemoterápica aos pacientes do HUPE.

Na terceira semana de março de 2020, a primeira de isolamento social no Rio de Janeiro, houve necessidade de cancelamento de cirurgias no HUPE, devido ao baixo estoque de sangue $\mathrm{Rh}$ negativo e do tipo O positivo. Na mesma época, as campanhas de vacinação contra o sarampo e, em seguida, contra influenza, reforçaram causas de inaptidão temporária à doação, sendo mais um fator limitante e uma ameaça para uma eficiente promoção à doação.

No mundo, começaram a surgir orientações de diversas organizações, como da American Association of Blood Banks (AABB), da International Society of Blood Transfusion (ISBT), do Food and Drug Administration (FDA), da Associação Brasileira de Hematologia, Hemoterapia e Terapia Celular $(\mathrm{ABHH})$ e do Ministério da Saúde brasileiro, ressaltando a importância da manutenção de estoques de sangue. Vários hemocentros do país fizeram apelos para que o 
estoque de hemocomponentes fosse mantido, priorizando o respeito ao distanciamento social, sem prejuízo da proteção aos doadores, que precisaram sair de casa para efetuar sua doação. No caso do NHHUPE, em diversas chamadas para doadores, foi ressaltado que aqueles com mais de 60 anos poderiam permanecer em casa, a fim de evitar a exposição comunitária ao SARS-Cov-2, uma vez que, de acordo com dados da literatura, são mais vulneráveis à COVID-19.

Baseados nas orientações de segurança transfusional, à época a Nota Técnica n.o 5/2020-CGSH-DAET/SAES/MS ${ }^{(16)}$ e a de n.o 13/2020-CGSH-DAET/SAES/MS ${ }^{(10)}$, o NHHUPE estabeleceu estratégias que incluíam o agendamento de doações, proteção de colaboradores e doadores com medidas de distanciamento social, higiene das mãos com água e sabão ou álcool em gel, observância das regras de etiqueta respiratória, rigorosa higienização de ambientes e equipamentos, uso de EPI pelos profissionais da saúde e orientações aos doadores para que entrassem em contato caso apresentassem sintomas gripais nos 14 dias seguintes à doação.

Para contornar o cenário desfavorável, a equipe de captação do NHHUPE intensificou a abordagem via mídias sociais, e-mails e telefonemas para doadores e órgãos públicos, como a Guarda Municipal do Município do Rio de Janeiro, e obteve importante e significa- tiva parceria das assessorias de comunicação do HUPE, da UERJ (COMUNS, TV UERJ, e do projeto de extensão universitária da Faculdade de Enfermagem, cujo resultado foi percebido na retomada das doações, com pico no dia 20 de março, mantendo a média de doações neste mês acima da média histórica (Figura 3). Estas ações não acrescentaram despesa para o serviço, uma vez que envolveram a força de trabalho de pessoas regularmente envolvidas no processo; todavia, trouxe à tona a necessidade de plano de contingência para tempos de crise.

\section{CONCLUSÃO}

Quando pensamos em abastecimento de hemocomponentes, questionamos: Quem são os doadores? Como eles estão? Como chegarão até nós, ou como chegaremos até eles?

Durante uma pandemia, quando quarentena e distanciamento social são propostas que podem salvar vidas, medidas que primem pela segurança do doador, do receptor e dos profissionais da saúde devem ser reforçadas e discutidas amplamente entre a equipe. Abordagens sobre o uso racional do sangue ou "patient blood management” são mais que nunca necessárias e devem ser o foco do hemoterapeuta junto ao corpo clínico e cirúrgico, assim como o compartilhamento de estratégias para manutenção de estoques e da segurança transfusional. Estudos avaliando o impacto da COVID-19 na demanda transfusional e nas ações de hemovigilância voltadas a estes pacientes, poderão esclarecer muito sobre o papel das transfusões e possíveis reações transfusionais na vigência da "tempestade inflamatória" descrita em outros artigos ${ }^{(17)}$. A hemoterapia poderá se envolver nas estratégias para combate à COVID-19, através da obtenção de plasma imune para infusão passiva de anticorpos neutralizantes ${ }^{(18-21)} \mathrm{e}$, ainda, numa eventual possibilidade de uso de plasmaférese nos casos graves, com intensa atividade inflamatória, caso haja evidência de sua eficácia ${ }^{(22,23)}$.

Diante de uma nova doença, de caráter pandêmico, temos mais perguntas que respostas, porém enquanto não houver substituto para o sangue, a captação de doadores e a capacidade de adaptação a situações adversas poderá fazer a diferença. Sugerimos que mais e mais centros compartilhem suas estratégias para a garantia de sangue em momentos de crise.

\section{AGRADECIMENTOS}

À Enfermeira Barbara Suzana Silva, Especialista em Enfermagem Pediátrica e Neonatologia e em Vigilância Sanitária em Serviços de Saúde da Universidade do Estado do Rio de Janeiro; e à Médica Marcia Babo, Hematologista e Hemoterapeuta da Universidade do Estado do Rio de Janeiro.

\section{REFERÊNCIAS}

1. Qun L, Guan X, Wu P, Wang X, Zhou L, Tong $Y$, et al. Early Transmission Dynamics in Wuhan, China, of Novel Coronavirus-Infected Pneumonia. N Engl J Med [Internet]. 2020 Mar 24; 382:1199-1207. doi: http://doi.org/10.1056/nejmoa2001316.

2. Wu Ym Chen C, Chan Y. The outbreak of COVID-19: An overview. Journal of the Chinese Medical Association [Internet]. 2020; 83(3):217-220. doi: https://doi.org/10.1097/ JCMA.0000000000000270.

3. WHO Director-General's opening remarks at the media briefing on COVID-19 [Internet]. Genebra: World Health Organization; 2020 [citado em 10 abr 2020]. Disponivel em: https://www. who.int/dg/speeches/detail/who-director-general-s-opening-remarks-at-the-media-briefing-on-covid-19---11-march-2020.

4. Rio de Janeiro (Estado). Decreto n. ${ }^{\circ}$ 46.966, de 11 de março de 2020. Dispõe sobre as medidas para enfrentamento da emergência de saúde pública de importância internacional de- corrente do coronavírus, e dá outras providências. Diário Oficial do Estado do Rio de Janeiro [Internet]: parte 1: Poder Executivo, Rio de Janeiro, 46(45-A):1 [citado em 10 abr 2020]. Disponível em: http://www.ioerj.com.br/portal/modules/conteudoonline/ mostra_edicao.php?session=VFhwVkOwNXJSa1pOUkdkMFVYcEdRMDVUTURCT2FsVXpURIZGTIU1NINYUk5hMFpDVFhwcmQwMXJUWGROZW1NeQ==\&p=MQ==\&tb=RGVjcmVObyBOwrogNDY5NjYmlzAxMzs=.

5. Ministério da Saúde (BR). Portaria n. ${ }^{\circ}$ 428, de 19 de março de 2020. Dispõe sobre as medidas de proteção para enfrentamento da emergência de saúde pública de importância internacional decorrente do coronavírus (covid-19) no âmbito das unidades do Ministério da Saúde no Distrito Federal e nos Estados. Diário Oficial da União. [Internet]. 20 mar 2020; Seção 55(1):149 [citado em 10 abr 2020]. Disponivel em: http://www.in.gov.br/en/web/ dou/-/portaria-n-428-de-19-de-marco-de-2020-249027772.

6. Agência Nacional de Vigilância Sanitária (BR). Nota técnica GVIMS/GGTES/ANVISA n. ${ }^{\circ}$ 04/2020. Orientações para serviços 


\section{REFERÊNCIAS}

de saúde: medidas de prevenção e controle que devem ser adotadas durante a assistência aos casos suspeitos ou confirmados de infecção pelo novo coronavírus (SARS-CoV-2). [Internet]. Brasília, DF: Anvisa, 2020 [atualizada em 21 mar 2020; [citado em 07 abr 2020]. 73 p. Disponivel em: http://portal.anvisa. gov.br/documents/33852/271858/Nota+T\%C3\%A9cnica+n+04-2020+GVIMS-GGTES-ANVISA/ab598660-3de4-4f14-8e6f-b9341c196b28.

7. Ministério da Saúde, Secretaria de Vigilância em Saúde (BR). Plano de Contingência Nacional para Infecção Humana pelo novo Coronavírus COVID-19. [Internet]. Brasília, DF: Centro de Operações de Emergências em Saúde Pública COVID-19, fev 2020. 24 p [citado em 07 abr 2020]. Disponivel em: https://portalarquivos2.saude.gov.br/images/pdf/2020/fevereiro/13/plano-contingencia-coronavirus-COVID19.pdf.

8. Rio de Janeiro (Estado). Secretaria Estadual de Saúde. Nota técnica SVS/SES-RJ nº8/2020. Doença pelo Coronavírus (COVID-19). [Internet]. Rio de Janeiro: Subsecretaria de Vigilância em Saúde, 2020 [atualizada em 18 mar 2020; [citado em 08 abr 2020]. 7 p. Disponivel em: https://www.saude.rj.gov.br/comum/ code/MostrarArquivo.php?C=Mjk3Njc\%2C.

9. Rio de Janeiro (Estado). Secretaria Estadual de Saúde. Nota técnica SVS/SES-RJ n. ${ }^{\circ}$ 09/2020. Doença pelo Coronavírus (COVID-19). [Internet]. Rio de Janeiro: Subsecretaria de Vigilância em Saúde, 2020. [atualizada em 24 mar 2020]; [citado em 07 abr 2020]. 2 p. Disponivel em: https://www.saude.rj.gov.br/comum/code/MostrarArquivo.php?C=Mjk5NDE\%2C.

10. Ministério da Saúde, Departamento de Atenção Especializada e Temática, Coordenação Geral de Sangue e Hemoderivados (BR). Nota técnica n. ${ }^{\circ}$ 13/2020-CGSH/DAET/SAES/MS. Atualização dos critérios técnicos contidos na Nota Técnica N 5 /2020CGSH/DAET/SAES/MS para triagem clínica dos candidatos à doação de sangue relacionados ao risco de infecção pelo SARS-CoV-2, (vírus causador da COVID-19). [Internet]. Rio de Janeiro: CGSH, 2020. [atualizado em: 27 mar 2020]; [citado em: 9 abr 2020]. 4 p. Disponível em: https://www.saude.gov.br/images/ pdf/2020/marco/27/SEI-MS---0014052636---Nota-T--cnica--n---13-2020.pdf.

11. Johns Hopkins University. Center for Systems Science and Engineering [Internet]. Coronavirus COVID-19: global cases [Internet]. 2020 [citado em 24 mar 2020]. Disponivel em: https:// gisanddata.maps.arcgis.com/apps/opsdashboard/index.html\#/ bda7594740fd40299423467b48e9ecf6.

12. Ministério da Saúde, Secretaria de Vigilância em Saúde (BR). Boletim Epidemiológico [Internet]. Brasília, DF: Secretaria de Vigilância em Saúde. N. 7, abr 2020 com título: Especial Doença pelo Coronavírus 2019. [citado em 7 abr 2020] Disponível em: https://www.saude.gov.br/images/pdf/2020/April/06/ 2020-04-06---BE7---Boletim-Especial-do-COE---Atualizacao-da-Avaliacao-de-Risco.pdf.

13. Universidade do Estado do Rio de Janeiro. Faculdade de Enfermagem. Projeto Sangue: vencendo o medo garantindo a vida [Internet]. Rio de Janeiro, 2020. [citado em 07 abr 2020]. Dispo- nível em: https://www.facebook.com/Projeto-Sangue-vencendo-o-medo-garantindo-a-Vida-451682228188410/.

14. Universidade do Estado do Rio de Janeiro. Atenção: Uerj prorroga suspensão de atividades não essenciais por mais 15 dias [Internet]. Rio de Janeiro, 2020. [citado 16 mar 2020]. Disponível em: https://www.uerj.br/noticia/10477/.

15. Universidade do Estado do Rio de Janeiro. Reitoria. Ato Executivo de Decisão Administrativa n. ${ }^{\circ}$ 013/2020, de 16 de março de 2020. Regulamenta o Decreto . 46.970, de 13 de março de 2020 e a Resolução Conjunta SCTI/UERJ n. ${ }^{\circ}$ 9, de 13 de março de 2020, que tratam das medidas temporárias de prevenção ao contágio e de enfrentamento da propagação decorrente do novo coronavírus (COVID-19) [Internet]. Rio de Janeiro, 2020. [citado em 08 abr 2020]. Disponível em: http://www.boluerj.uerj.br/pdf/ aeda_00132020_16032020.pdf.

16. Ministério da Saúde, Departamento de Atenção Especializada e Temática, Coordenação-Geral de Sangue e Hemoderivados (BR). Nota técnica n. ${ }^{\circ}$ 5/2020-CGSH/DAET/SAES/MS. Atualização dos critérios técnicos para triagem clínica de dengue (DENV), chikungunya (CHIKV), zika (ZIKV) e coronavírus (SARS, MERS, 2019-nCoV) nos candidatos à doação de sangue. [Internet]. Rio de Janeiro: CGSH, 2020. [atualizado em 21 fev 2020]; [citado em 08 abr 2020]. 5 p. Disponível em: https://www.saude.gov.br/ images/pdf/2020/fevereiro/21/SEI-MS---0013484477---Nota-T--cnica.pdf.

17. Mehta P, McAuley DF, Brown M, Sanchez E, Tatersall RS, Manson JJ, et al. COVID-19: consider cytokine storm syndromes and immunosuppression. Lancet. [Internet]. $2020 \mathrm{Mar}$ 28 395(10229):1011-1088, e54-e61. doi:10.1016/S01406736(20)30628-0.

18. Duan K, Liu B, Li C, Zhang H, Yu T, Qu J, et al. Effectiveness of convalescent plasma therapy in severe COVID-19 patients. Proc Natl Acad Sci U S A [Internet]. 2020 Apr 6. doi: 10.1073/ pnas.2004168117.

19. Chen L, Xiong J, Bao L, Shi Y. Convalescent plasma as a potential therapy for COVID-19. Lancet Infect Dis. [Internet]. 2020 Apr 1; 20(4):398-400. doi:10.1016/ S1473-3099(20)30141-9.

20. Roback JD, Guarner J. Convalescent plasma to treat COVID-19: possibilities and challenges. JAMA [Internet]. 2020 Mar 27. doi:10.1001/jama.2020.4940.

21. Shen C, Wang Z, Zhao F, Yang Y, Li J, Yuan J, et al. Treatment of 5 critically ill patients with COVID-19 with convalescent plasma. JAMA. [Internet]. 2020 Mar 27. doi: 10.1001/jama.2020.4783.

22. Keith P, Day M, Perkins L, Moyer L, Hewitt K, Wells A. A novel treatment approach to the novel coronavirus: an argument for the use of therapeutic plasma exchange for fulminant COVID-19. Crit Care. [Internet]. 2020 Apr 2; 24(128). doi:10.1186/s13054020-2836-4.

23. Ma J, Xia P, Zhou Y, Liu Z, Zhou X, Wang J, et al. Potential effect of blood purification therapy in reducing cytokine storm as a late complication of critically ill COVID-19. Clin Immunol. $2020 \mathrm{Apr}$ 1;214; 108408. doi:10.1016/j.clim.2020.108408 